\title{
The prognostic power of inflammatory indices and clinical factors in metastatic castration-resistant prostate cancer patients treated with radium-223 (BIO-Ra study)
}

\author{
Matteo Bauckneht ${ }^{1,2}$ (1) . Sara Elena Rebuzzi $i^{3,4}$ - Alessio Signori ${ }^{2} \cdot$ Viviana Frantellizzi $^{5} \cdot$ Veronica Murianni $^{3,4}$. \\ Elisa Lodi Rizzini ${ }^{6} \cdot$ Manlio Mascia $^{7}$. Valentina Lavelli ${ }^{8}$. Maria Isabella Donegani ${ }^{1,2} \cdot$ Marta Ponzano $^{2}$. \\ Angela Gaudiano $^{8} \cdot$ Maria Lina Stazza $^{9} \cdot$ Maria Licari $^{10}$ - Letizia Cavallini ${ }^{6,11}$. Viola Laghi ${ }^{6,11} \cdot$ Luca Cindolo $^{12}$. \\ Martina Maggi ${ }^{13}$. Alessandro Sciarra ${ }^{13}$. Paolo Mammucci ${ }^{8}$. Gianmario Sambuceti ${ }^{1,2}$. Renato Patrizio Costa ${ }^{10}$. \\ Angela Spanu ${ }^{9} \cdot$ Giuseppe Rubini $^{8} \cdot$ Fabio Monari $^{6}$. Giuseppe De Vincentis ${ }^{5}$. Giuseppe Fornarini ${ }^{3}$
}

Received: 3 June 2021 / Accepted: 29 August 2021 / Published online: 6 September 2021

(c) The Author(s) 2021

\begin{abstract}
Purpose To combine peripheral blood indices and clinical factors in a prognostic score for metastatic castration-resistant prostate cancer (mCRPC) patients treated with radium-223 dichloride ([ $\left.\left.{ }^{223} \mathrm{Ra}\right] \mathrm{RaCl}_{2}\right)$.

Patients and methods Baseline neutrophil-to-lymphocyte ratio (NLR), derived NLR (donor), lymphocyte-to-monocyte ratio (LMR), platelet-to-lymphocyte ratio (PLR), systemic inflammation index (SII), Eastern Cooperative Oncology Group performance status (ECOG PS), Gleason score (GS) group, number of bone metastases, prostate-specific antigen (PSA), alkaline phosphatase (ALP), line of therapy, previous chemotherapy, and the presence of lymphadenopathies were collected from seven Italian centers between 2013 and 2020. Lab and clinical data were assessed in correlation with the overall survival (OS). Inflammatory indices were then included separately in the multivariable analyses with the prognostic clinical factors. The model with the highest discriminative ability (c-index) was chosen to develop the BIO-Ra score.

Results Five hundred and nineteen mCRPC patients (median OS: 19.9 months) were enrolled. Higher NLR, dNLR, PLR, and SII and lower LMR predicted worse OS (all with a $p<0.001$ ). The multivariable model including NLR, ECOG PS, number of bone metastases, ALP, and PSA (c-index: 0.724) was chosen to develop the BIO-Ra score. Using the Schneeweiss scoring system, the BIO-Ra score identified three prognostic groups (36\%, 27.3\%, and $36.6 \%$ patients, respectively) with distinct median OS (31, 26.6, and 9.6 months, respectively; hazard ratio: $1.62, p=0.008$ for group 2 vs. 1 and 5.77, $p<0.001$ for group 3 vs. 1$)$.

Conclusions The BIO-Ra score represents an easy and widely applicable tool for the prognostic stratification of mCRPC patients treated with $\left[{ }^{223} \mathrm{Ra}\right] \mathrm{RaCl}_{2}$ with no additional costs.
\end{abstract}

Keywords Metastatic castration-resistant prostate cancer $\cdot\left[{ }^{223} \mathrm{Ra}^{2} \mathrm{RaCl}_{2} \cdot\right.$ Inflammatory indices $\cdot$ Neutrophil-to-lymphocyte ratio $\cdot$ Clinical factors

Matteo Bauckneht and Sara Elena Rebuzzi contributed equally to this work.

This article is part of the Topical Collection on Oncology Genitourinary

Matteo Bauckneht

matteo.bauckneht@hsanmartino.it

Extended author information available on the last page of the article

\section{Introduction}

Bone metastases represent the leading cause of poor quality of life and increased mortality in patients with metastatic castration-resistant prostate cancer (mCRPC) $[1,2]$. Radium-223 dichloride $\left(\left[{ }^{223} \mathrm{Ra} \mathrm{RaCl}_{2}\right)\right.$ is an alpha-emitting radioisotope that selectively binds to increased osteoblastic activity areas, inducing doublestranded DNA breaks impairing cellular repair mechanisms [3]. 
The phase III Alpharadin in Symptomatic Prostate Cancer Patients (ALSYMPCA) study demonstrated a significantly prolonged overall survival (OS) and time to first symptomatic skeletal event in mCRPC patients with bone metastasis and no visceral metastatic involvement receiving [ $\left.{ }^{223} \mathrm{Ra}\right]$ $\mathrm{RaCl}_{2}$ compared to placebo [4]. According to these results, $\left[{ }^{223} \mathrm{Ra}\right] \mathrm{RaCl}_{2}$ was approved by the Food and Drug Administration in 2013 [5].

However, in later years, the clinical experience has revealed a lower survival benefit than ALSYMPCA results. Several studies reported a median OS (mOS) ranging from 6 to 10 months, which was lower than that reported by the ALSYMPCA trial (14.9 months) [6-8]. Previous studies suggested that this is partially due to a suboptimal selection of patients with unfavorable prognostic characteristics [9]. Moreover, in 2018 the European Medicine Agency (EMA) restricted the use of $\left[{ }^{223} \mathrm{Ra}\right] \mathrm{RaCl}_{2}$ to patients with more than six osteoblastic lesions progressing to at least two systemic therapies for mCRPC or ineligible for any systemic mCRPC treatment [10]. The consequent delay of $\left[{ }^{223} \mathrm{Ra}\right] \mathrm{RaCl}_{2}$ administration in the later phases of the disease might negatively affect OS, making the patient's selection process even further challenging [11]. In this scenario, identifying prognostic factors potentially able to select patients most likely to benefit from $\left[{ }^{223} \mathrm{Ra}\right] \mathrm{RaCl}_{2}$ since baseline has become a crucial issue.

Cancer-associated inflammation plays a key role in therapeutic response and survival in cancer patients across different tumor types [12]. Many research groups have investigated the predictive and prognostic role of peripheral blood inflammatory parameters, such as the neutrophil-to-lymphocyte ratio (NLR), in different solid tumors [13-17].

A preliminary monocentric study investigated the prognostic role of baseline peripheral blood inflammatory indices focusing on mCRPC patients treated with $\left[{ }^{223} \mathrm{Ra}\right] \mathrm{RaCl}_{2}$ showing that increased NLR at baseline identifies patients at higher risk for unfavorable outcomes [18]. With the BIORa study, we extended this analysis to a multicentric setting, aiming to develop a composite prognostic score potentially able to improve the patients' selection process.

\section{Materials and methods}

The study was performed according to the Declaration of Helsinki, Good Clinical Practice, and local ethical regulations. The local ethical committee of the leading center approved the study (Regional Ethical Committee of Liguria-registration number 535/2020). The study was then approved by the local ethical committee of each adhering center. All patients enrolled in the study signed written informed consent before each $\left[{ }^{223} \mathrm{Ra}^{2} \mathrm{RaCl}_{2}\right.$ administration, which included the use of anonymized data for retrospective research purposes.

\section{Study population}

A multicenter analysis was conducted on seven Italian centers retrospectively collecting clinical data and laboratory parameters of mCRPC patients receiving $\left[{ }^{223} \mathrm{Ra}^{2} \mathrm{RaCl}_{2}\right.$ in a real-world setting. CRPC was defined as a serum testosterone level of $<50 \mathrm{ng} / \mathrm{dL}$ following surgical or pharmaceutical castration. According to the standard selection criteria for $\left.{ }^{223}{ }^{22 a}\right] \mathrm{RaCl}_{2}$ treatment, patients must have a diagnosis of mCRPC with symptomatic bone metastases and neither visceral metastases nor lymph nodes $>3 \mathrm{~cm}$ in short-axis diameter [19]. To be included in the study, patients must have received at least one cycle of $\left[{ }^{223} \mathrm{Ra}\right] \mathrm{RaCl}_{2}$.

\section{Treatment}

$\left[{ }^{223} \mathrm{Ra}\right] \mathrm{RaCl}_{2}(50-55 \mathrm{KBq} / \mathrm{kg})$ was intravenously administrated every 4 weeks and was continued until disease progression, death, or patient choice up to six cycles. Eventual toxicities were managed according to the current guidelines [19]. Treatment with either chemotherapy, abiraterone, or enzalutamide was discontinued before the first $\left[{ }^{223} \mathrm{Ra}\right] \mathrm{RaCl}_{2}$ administration. Patients continued receiving androgen deprivation therapy [19].

\section{Systemic inflammation indices}

Complete blood count as assessed soon before the first $\left[{ }^{223} \mathrm{Ra}\right] \mathrm{RaCl}_{2}$ administration collecting white blood cells (WBC), platelets (PLT) and the absolute neutrophil (ANC), lymphocyte (ALC), and monocyte (AMC) count. From the complete blood count, we calculated the following ratios: NLR, derived NLR (dNLR), lymphocyteto-monocyte ratio (LMR), platelets-to-lymphocyte ratio (PLR), and systemic inflammation index (SII). dNLR was calculated as ANC/(WBC-ANC) and SII as NLRxPLT.

\section{Cut-off determination of the systemic inflammation indices}

As many thresholds have been explored, but none validated in $\mathrm{mCRPC}$ patients (especially in those treated with $\left[{ }^{223} \mathrm{Ra}\right]$ $\mathrm{RaCl}_{2}$ ), the cut-off values of inflammatory indices were determined using time-dependent ROC curves with the Liu approach, maximizing the concordance probability function [20-23]. The ROC curve was calculated at the time point corresponding to the mOS. An internal validation using 
500-times bootstrap resampling was performed. For each index, the optimal cut-off and the c-index were reported.

\section{Study endpoint}

The primary endpoint was overall survival (OS), which was defined as the time from first $\left[{ }^{223} \mathrm{Ra}\right] \mathrm{RaCl}_{2}$ administration until death from any cause, censored at last follow-up for patients who were alive.

\section{Statistical analyses}

The descriptive analyses were conducted using absolute frequency and percentage for categorical variables and by median and range for quantitative variables. The Kaplan-Meier (KM) method was used to estimate the survival curve of OS [24]. Differences were considered statistically significant when the $p$-value $(p)$ was $<0.05$. Univariable and multivariable analyses were performed using Cox proportional hazard regression model, estimating hazard ratios (HRs), and their $95 \%$ confidence interval (CI). In the univariable analyses, clinical and laboratory parameters were assessed in correlation with OS. Only factors with a $p<0.05$ at the univariable analysis were evaluated in the multivariable analyses for OS. Due to a strict correlation among the inflammatory indices, those predictive of OS were included separately in the multivariable analyses together with the clinical factors. Only factors with a $p<0.05$ in the multivariable analysis were kept in the multivariable model. For each multivariable model, the discriminatory ability as defined by Harrell's c-index was calculated: a higher c-index represented a better capability of the multivariable model to separate patients with and without the event. A 500-times bootstrap resampling with replacement guaranteed the stability of the c-index. Missing values for indices were imputed (see Supplementary Materials for details). The multivariable model with the highest c-index was chosen for being the basis of the prognostic score. All statistical analyses were performed using the software Stata v.16 (StataCorp 2019) and R v.4.0.2 [25, 26].

\section{Prognostic score}

The selection procedure for the prognostic score and the parameter estimation from the Cox model was internally validated using the bootstrap approach (see Supplementary Materials for details).

To consider the possible overfitting during building and estimation of the prognostic score, a bias-corrected estimate of the discriminatory ability (c-index) was calculated with 500 bootstrap samples using the Design package in $\mathrm{R}$.

The prognostic score was calculated using the regression coefficient based (Schneeweiss) scoring system. The weight assigned to each factor in the score was defined based on the Cox regression model's regression coefficient [27].

Finally, the prognostic score was stratified in risk strata according to the likelihood-ratio test and after checking the score's survival estimates.

\section{Dynamic alkaline phosphatase (ALP) change}

The association of each determinant included in the BIO$\mathrm{Ra}$ score and the score itself with the dynamic alkaline phosphatase (ALP) change was investigated using a linear regression model with the dynamic ALP change as the dependent variable. Dynamic ALP change was defined as the percentage change at week 12 , after the third cycle of [223Ra] $\mathrm{RaCl}_{2}$, the halfway point of treatment, as previously described [28]. Results were reported as linear regression coefficients with $95 \%$ confidence intervals.

\section{Results}

\section{Patients' characteristics}

Five hundred and nineteen mCRPC patients were included in the analysis. Patients' and treatment characteristics are summarized in Table 1. The median age was 74 years (range: $50-90$ years), and patients with $\geq 75$ years were $48 \%$ of the entire cohort. At the time of $\left[{ }^{223} \mathrm{Ra}\right] \mathrm{RaCl}_{2}$ initiation, most patients had an Eastern Cooperative Oncology Group performance status (ECOG PS) of 0-1 (77\%), no lymph nodal metastases (61\%), and a number of bone metastases between 6 and $20(57 \%)$. Among all patients, $48 \%$ received $\left[{ }^{223} \mathrm{Ra}\right]$ $\mathrm{RaCl}_{2}$ as first- and second-line therapy, while $52 \%$ as further lines, while most of them had previously received chemotherapy (62\%).

\section{Results in the overall population}

At the time of data cut-off (February 2021), with a median follow-up of 10.7 months, 251 patients (48\%) were dead and the mOS was 19.9 months (95\% CI 17.7-23.8), and the completion rates of the first three and six cycles were $88 \%$ and $63 \%$, respectively.

\section{Identification of the cut-off values}

Bone scan lesions were categorized in $<6,6-20$, and $>20$, as previously described [29]. According to the current guidelines [19], patients with advanced diffuse metastatic 
Table 1 Patients' characteristics

\begin{tabular}{ll}
\hline Patients $n=519$ & \\
Characteristics & $N(\%)$ \\
\hline
\end{tabular}

Age, years

Median (range) $74(50-90)$

$<75$

268 (52)

$\geq 75$

Gleason score

Median (range)

$\leq 7$

$\geq 8$

Missing

ECOG PS

Median (range)

0-1

2-3

$\left[{ }^{223} \mathrm{Ra}\right] \mathrm{RaCl}_{2}$ treatment line

Median (range)

First and second line

$\geq$ Third line

251 (48)

8 (5-10)

173 (33)

263 (51)

83 (16)

$1(0-3)$

399 (77)

120 (23)

$3(1-9)$

249 (48)

$270(52)$

Prior chemotherapy

Yes

324 (62)

No

195 (38)

Lymphadenopathies

Yes

$161(31)$

No

Missing

$\mathrm{N}$ bone metastases

$<6$
$6-20$
$>20$

Missing

Bisphosphonates/denosumab use

Yes

No

Missing

317 (61)

$41(8)$

$60(12)$

$295(57)$

155 (30)

9 (2)

233 (45)

283 (55)

3 (1)

$\left[{ }^{223} \mathrm{Ra}\right] \mathrm{RaCl}_{2}$ treatment before/after EMA restriction
After
98 (19)
Before
421 (81)

Baseline ALP, U/L

Median (range)

$<220$

$\geq 220$

Missing

Baseline median LDH, U/L (range)

Baseline median PSA, ng/ml (range)

Baseline median $\mathrm{Hb}, \mathrm{g} / \mathrm{dL}$ (range)

$142(0-2474)$

340 (66)

167 (32)

12 (2)

294 (129-2146)

$54.0(0.03-6089)$

$12.2(7.8-16.4)$

ECOG Eastern Cooperative Oncology Group, EMA European Medicines Agency, $A L P$ alkaline phosphatase, $L D H$ lactate dehydrogenase, $P S A$ prostate-specific antigen, $H b$ hemoglobin
Table 2 Univariable Cox regression analyses

\begin{tabular}{|c|c|c|c|}
\hline & $N$ & $H R(95 \% C I)$ & $p$ value \\
\hline NLR & 392 & & $<0.001$ \\
\hline$<3.1$ & 263 & 1.00 (ref) & \\
\hline$\geq 3.1$ & 129 & $3.14(2.26-4.38)$ & \\
\hline dNLR & 423 & & $<0.001$ \\
\hline$<2.0$ & 257 & 1.00 (ref) & \\
\hline$\geq 2.0$ & 166 & $2.09(1.52-2.85)$ & \\
\hline LMR & 390 & & $<0.001$ \\
\hline$<2.8$ & 123 & 1.00 (ref) & \\
\hline$\geq 2.8$ & 267 & $0.54(0.39-0.75)$ & \\
\hline PLR & 393 & & $<0.001$ \\
\hline$<145.9$ & 194 & 1.00 (ref) & \\
\hline$\geq 145.9$ & 199 & $2.03(1.49-2.78)$ & \\
\hline SII & 392 & & $<0.001$ \\
\hline$<768.8$ & 260 & 1.00 (ref) & \\
\hline$\geq 768.8$ & 132 & $2.76(1.98-3.84)$ & \\
\hline ECOG PS & 519 & & $<0.001$ \\
\hline $0-1$ & 399 & 1.00 (ref) & \\
\hline $2-3$ & 120 & $1.76(1.30-2.38)$ & \\
\hline Gleason score group & 439 & & 0.189 \\
\hline$<8$ & 173 & 1.00 (ref) & \\
\hline$\geq 8$ & 266 & $0.84(0.64-1.09)$ & \\
\hline $\begin{array}{l}\mathbf{N} \\
\text { bone metastases }\end{array}$ & 510 & & \\
\hline$<6$ & 60 & 1.00 (ref) & \\
\hline $6-20$ & 295 & $1.42(0.92-2.20)$ & 0.116 \\
\hline$\geq 20$ & 155 & $3.23(2.06-5.07)$ & $<0.001$ \\
\hline ALP, U/L & 507 & & $<0.001$ \\
\hline$<220$ & 340 & 1.00 (ref) & \\
\hline$\geq 220$ & 167 & $2.42(1.83-3.21)$ & \\
\hline ALP, U/L & & & $<0.001$ \\
\hline$\leq 140$ & 249 & 1.00 (ref) & \\
\hline$>140$ & 258 & $1.95(1.51-2.53)$ & \\
\hline$\left[{ }^{223} \mathrm{Ra} \mathrm{RaCl}_{2}\right.$ therapy line & 519 & & 0.021 \\
\hline $1-2$ & 251 & 1.00 (ref) & \\
\hline$\geq 3$ & 268 & $1.34(1.05-1.72)$ & \\
\hline Previous chemotherapy & 519 & & 0.002 \\
\hline No & 195 & 1.00 (ref) & \\
\hline Yes & 324 & $1.51(1.16-1.96)$ & \\
\hline PSA, ng/mL & 515 & & $<0.001$ \\
\hline$<44$ & 231 & 1.00 (ref) & \\
\hline$\geq 44$ & 284 & $1.99(1.54-2.57)$ & \\
\hline $\mathrm{Hb}, \mathrm{g} / \mathrm{dL}$ & 518 & & $<0.001$ \\
\hline$<10$ & 31 & 1.00 (ref) & \\
\hline$\geq 10$ & 487 & $0.38(0.23-0.62)$ & \\
\hline
\end{tabular}

$N R L$ neutrophil-lymphocyte ratio, $d N L R$ derived neutrophil-lymphocyte ratio, $L M R$ lymphocyte-to-monocyte ratio, $P L R$ platelet-tomonocyte ratio, SII systemic immune-inflammation index, ECOG Eastern Cooperative Oncology Group, ALP alkaline phosphatase, $P S A$ prostate-specific antigen, $H b$ hemoglobin

Bold indicates significant $p$ values 


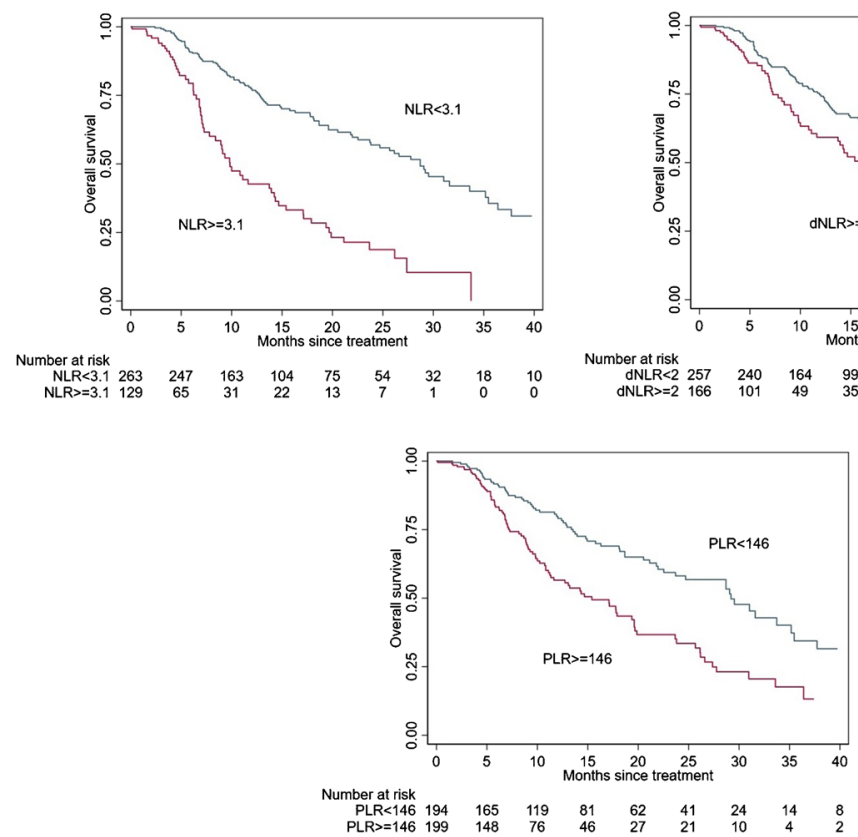

Fig. 1 Kaplan-Meier survival curves according to the systemic inflammatory indices. OS prediction according to neutrophil-to-lymphocyte ratio (NLR), derived-NLR (d-NLR), lymphocyte-to-mono-

infiltration of the bone ("superscan") should be treated with [223Ra] $\mathrm{RaCl}_{2}$ only after a careful benefit-risk assessment. Due to this subgroup's low statistical power, we included these patients in the third category. The cut-off values identified for the inflammatory indices were 3.1 for NLR, 2.0 for dNLR, 2.8 for LMR, 146 for PLR, and 769 for SII, while for prostate-specific antigen (PSA) was $44 \mathrm{ng} / \mathrm{mL}$. For each inflammatory index and PSA, the median value, the optimal cut-off, and the c-index are shown in Supplementary Table 1.

\section{Univariable analyses for OS}

Results from univariable analyses are reported in Table 2. All biomarkers and clinical factors, except for Gleason score (GS) group (defined as $<8$ or $\geq 8$ ) and the presence of lymphadenopathies, significantly correlated with OS at the univariable analyses (Table 2). Specifically, among inflammatory indices, higher NLR, dNLR, PLR, and SII were associated with worse OS, while higher LMR predicted longer OS (all with a $p<0.001$ ) (Fig. 1). The univariable analyses have been graphically summarized in one forest plot (Fig. 2).

\section{Multivariable analyses for OS}

Among 519 patients of the entire cohort, 494 patients (95\%) were evaluable for multivariable analyses after multiple imputations of missing inflammation indices. In the
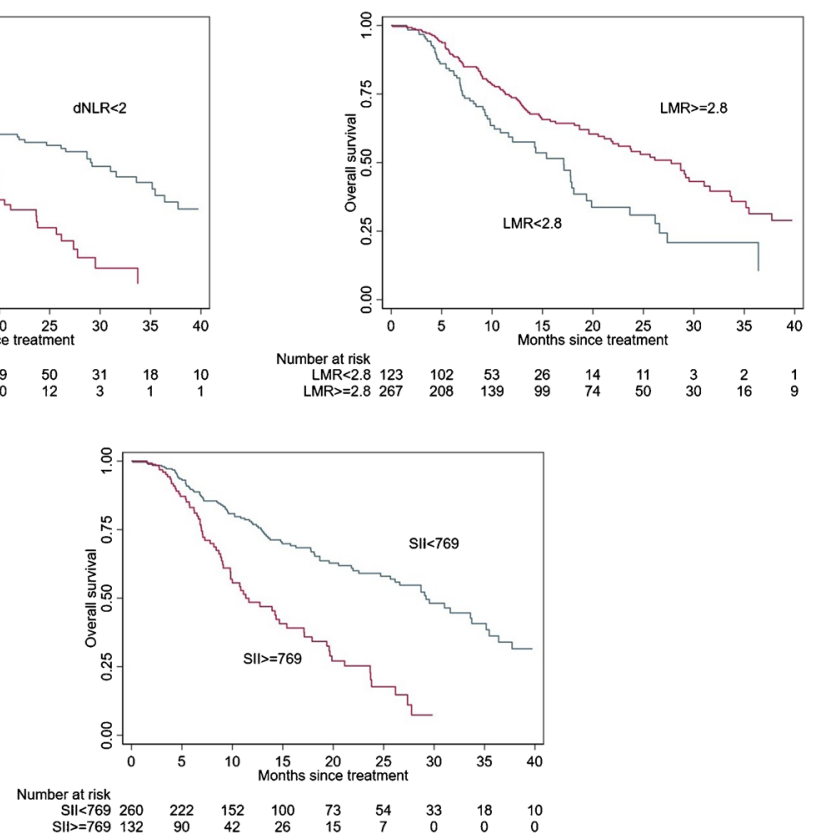

cyte ratio (LMR), platelets-to-lymphocyte ratio (PLR), and systemic inflammation index (SII)

multivariable analyses, all inflammatory indices, ECOG PS, the number of bone metastases, ALP, and PSA were confirmed as independent predictors of survival.

The multivariable model with $\operatorname{NLR}(<3.1$ vs. $\geq 3.1)$, ECOG PS (0-1 vs. 2-3), number of bone metastases $(<6$, 6-20,>20), ALP (<220 vs. $\geq 220)$, and PSA (<44 vs. $\geq 44)$ showed the highest discriminative ability (c-index: 0.724 ) (Table 3). For this reason, this multivariable model was chosen for the development of the prognostic score. Results were consistent with the analyses on complete cases (Supplementary Table 2).

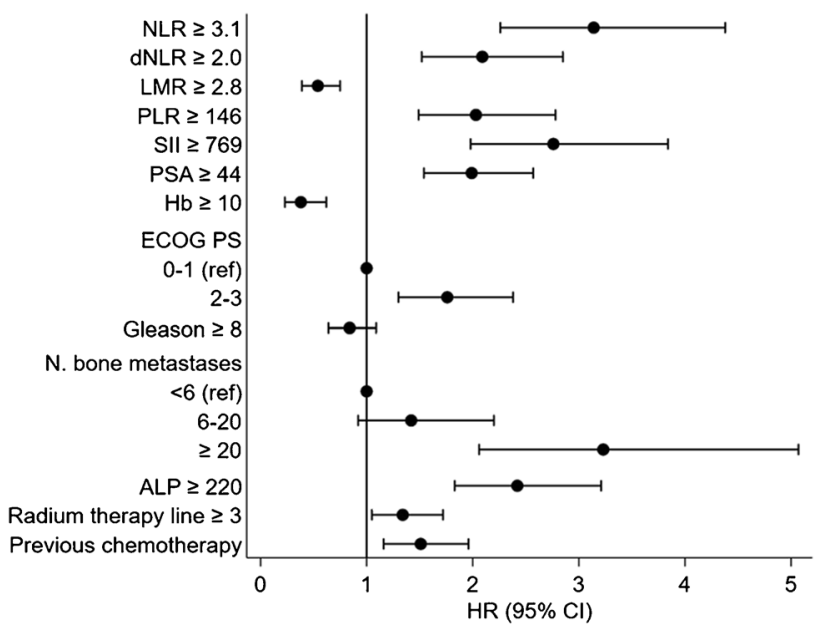

Fig. 2 Forest plot of the HRs obtained at the univariable analyses 


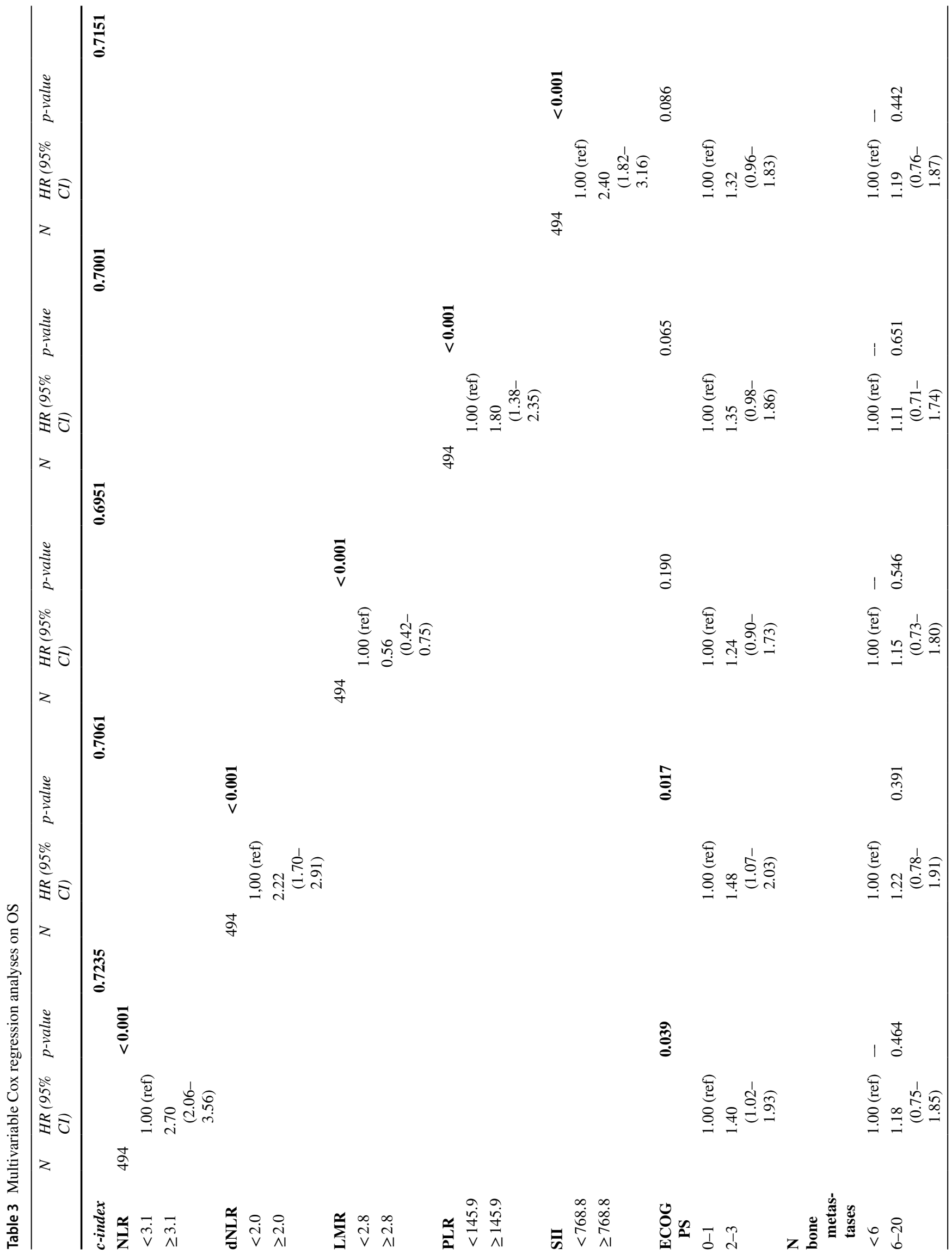




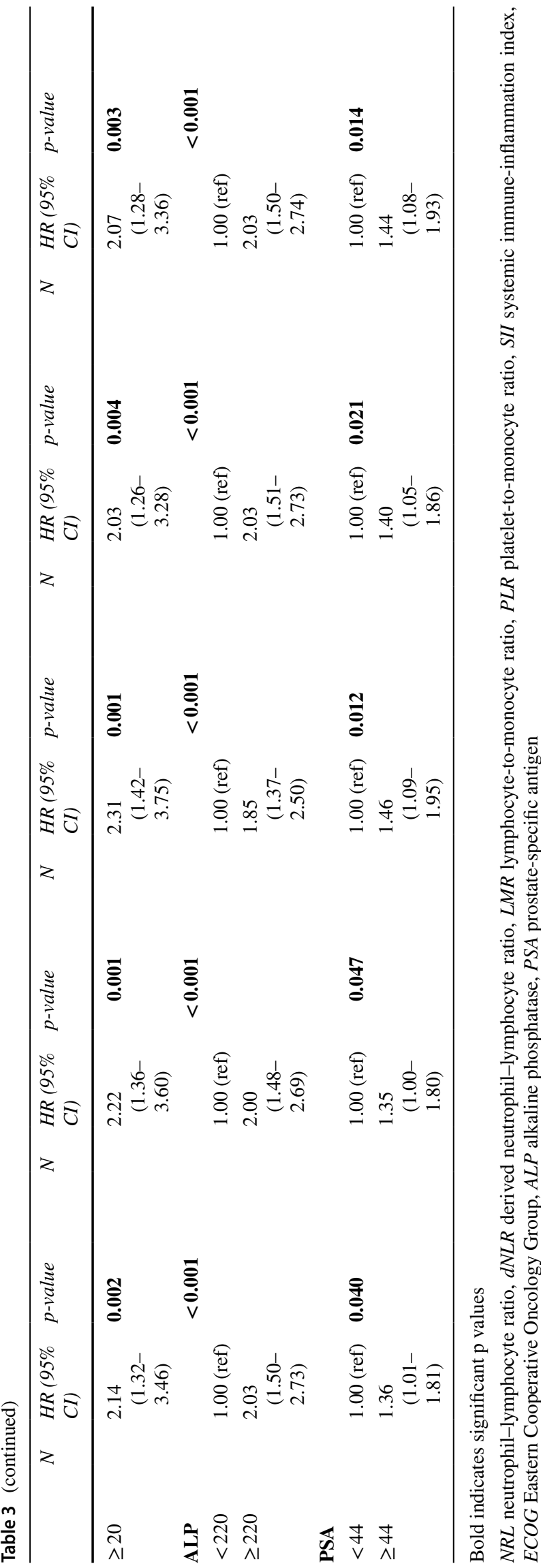

\section{Prognostic score}

After 500 bootstrap replications, in 276 replications (55.2\%), all five prognostic factors were included, while in $204(40.8 \%)$, four prognostic factors were maintained. The prognostic factors alternatively excluded were the PSA or the ECOG PS, while NLR, number of bone metastases, and ALP were kept in more than $95 \%$ of models. The regression parameters and HRs calculated from the 500 bootstrap samples were remarkably similar to those obtained from the original Cox model, suggesting an excellent internal validation (Table 4). The bias-corrected c-index for optimism from possible overfitting was 0.717 by the bootstrap procedure.

The point assignation according to the bootstrapped Cox model coefficients and the Schneeweiss scoring system was reported and ranged from a minimum of 0 to a maximum of 10 points. The final prognostic score, called "BIO-Ra score," had a c-index of 0.723. After application of the survival ROC curve and for a better clinical interpretation, the ten prognostic classes were combined in three prognostic groups characterized by very distinctive OS: the prognostic group 1 (score $0-2$ ), the prognostic group 2 (score 3-4), and the prognostic group 3 (score 5-10) (Fig. 3). According to the BIO-Ra score (Fig. 3), the prognostic group 1 (178 patients, 36\%) had a significantly longer mOS (31.0 months) compared to the prognostic group 2 (135 patients, 27.3\%) (26.6 months, HR 1.62, $p=0.008)$ and the prognostic group 3 (181 patients, $36.6 \%)$ (mOS: 9.6 months, $\mathrm{HR}=5.77, p<0.001$ ).

\section{Dynamic ALP change}

ALP values at baseline and at week 12 were both available in 383 patients. The mean ALP change was of $-7.6 \%$ (SD: 159; median:-23.1; IQR: - 41.7,-3.8). Neither of the determinants of the BIO-Ra score showed significant associations with the dynamic ALP change: NLR $(p=0.77)$, ECOG PS $(p=0.56)$, bone metastases (6-20 vs. $<6: p=0.39,>20$ vs. $<6, p=0.86)$, ALP $(p=0.092)$, and PSA $(p=0.17)$. Similarly, the three-level BIO-Ra score (3-4 and 5-10 vs. 0-2) was not associated with dynamic ALP change (coefficients of $-8.7,95 \% \mathrm{CI}=-47.8,30.4 ; p=0.66$, and $-16.2,95 \%$ $\mathrm{CI}=-54.4,22.1 ; p=0.41$, respectively).

\section{Discussion}

$\left[{ }^{223} \mathrm{Ra}\right] \mathrm{RaCl}_{2}$ is the first bone seeking radiopharmaceutical showing to both provide bone palliation and improve OS [4]. In the last years, it has become a valuable therapeutic option for mCRPC patients with symptomatic bone metastases. However, lower survival outcomes have been reported in real life [6], compared to the OS reported in the pivotal phase III ALSYMPCA trial [4]. 
This weaker survival benefit may be related to the lack of standardization of the optimal timing, sequence, and combinations of $\left[{ }^{223} \mathrm{Ra}\right] \mathrm{RaCl}_{2}$ with other therapeutic agents for mCRPC [9]. Moreover, there are no approved prognostic or predictive factors to identify mCRPC patients who would most benefit from $\left[{ }^{223} \mathrm{Ra} \mathrm{RaCl}_{2}\right.$ [30]. However, a growing amount of scientific data suggests that the efficacy of [ $\left.{ }^{223} \mathrm{Ra}\right]$ $\mathrm{RaCl}_{2}$ is strictly dependent on pre-treatment patients' prognostic stratification [9].

Several studies investigated many potential baseline prognostic factors, whose application might optimize the patient's selection process. In the present multicenter retrospective study, we observed that baseline ECOG PS, PSA and ALP levels, the number of bone metastases, and NLR provide prognostic insights in a large cohort of mCRPC patients receiving $\left[{ }^{223} \mathrm{Ra}^{2} \mathrm{RaCl}_{2}\right.$. These findings align with the literature since all these parameters represent established prognostic factors in this clinical setting. ECOG PS represents one of the most validated and reproducible tools for assessing the overall clinical status and one of the most important prognostic factors in treating advanced tumors, including mCRPC [31]. In fact, mCRPC patients treated with $\left[{ }^{223} \mathrm{Ra}\right] \mathrm{RaCl}_{2}$ show significantly longer survival outcomes in the presence of ECOG PS 0 than ECOG PS 1 or 2 [31]. Moreover, while the ALSYMPCA trial exclusively included patients with painful bone metastases [4], more recent data showed that OS tended to be longer in patients with little or no bone pain than those with moderate or severe pain [31].

On the other hand, many studies already showed the prognostic value of circulating PSA and ALP at baseline, as biochemical indicators of the tumor extent [28, 31-38]. This is coherent with the unfavorable prognosis observed in patients with massive bone metastases $(>20)$ at the bone scan or high tumor burden assessed with more sophisticated quantification approaches [32, 39-43].

Altogether, these data support the emerging notion that utilizing $\left[{ }^{223} \mathrm{Ra}\right] \mathrm{RaCl}_{2}$ early on in the course of mCRPC represents a reasonable and effective strategy [44]. Indeed, the administration of $\left[{ }^{223} \mathrm{Ra}\right] \mathrm{RaCl}_{2}$ before the ECOG PS declines to 2 or worse might increase the chances to complete the therapeutic scheme, which is crucial for deriving the maximal benefit from the therapy [4]. On the other hand, higher PSA or ALP values and higher bone metastases are related to a higher tumor burden, which carries a reduced response rate [42].
Table 4 Bootstrap internal validation of multivariable Cox regression coefficients and risk scoring definition

\begin{tabular}{|c|c|c|c|c|}
\hline & \multirow{2}{*}{$\begin{array}{l}\text { Original set }(\mathrm{N}=494) \\
\beta \text {-coefficient } \pm \mathrm{SE}\end{array}$} & \multicolumn{2}{|c|}{ Bootstrap (500 replication) } & \multirow{2}{*}{$\begin{array}{l}\text { Risk-scoring } \\
\text { (Schnee- } \\
\text { weiss) }\end{array}$} \\
\hline & & $\beta$-coefficient $\pm \mathrm{SE}$ & HR $(95 \% \mathrm{CI})$ & \\
\hline \multicolumn{5}{|l|}{ Parameter } \\
\hline \multicolumn{5}{|l|}{ NLR } \\
\hline$<3.1$ & 0 & 0 & 1.00 (ref) & 0 \\
\hline$\geq 3.1$ & $0.99 \pm 0.14$ & $1.02 \pm 0.15$ & $2.77(2.07-3.72)$ & 3 \\
\hline \multicolumn{5}{|l|}{ ECOG-PS } \\
\hline $0-1$ & 0 & 0 & 1.00 (ref) & 0 \\
\hline $2-3$ & $0.34 \pm 0.16$ & $0.35 \pm 0.18$ & $1.42(1.00-2.02)$ & 1 \\
\hline \multicolumn{5}{|l|}{$\mathrm{N}$ bone metastases } \\
\hline$<6$ & 0 & 0 & 1.00 (ref) & 0 \\
\hline $6-20$ & $0.17 \pm 0.23$ & $0.18 \pm 0.22$ & $1.20(0.78-1.84)$ & 1 \\
\hline$\geq 20$ & $0.76 \pm 0.25$ & $0.79 \pm 0.24$ & $2.20(1.38-3.53)$ & 3 \\
\hline \multicolumn{5}{|l|}{ ALP, U/L } \\
\hline$<220$ & 0 & 0 & 1.00 (ref) & 0 \\
\hline$\geq 220$ & $0.71 \pm 0.15$ & $0.71 \pm 0.16$ & $2.03(1.49-2.78)$ & 2 \\
\hline \multicolumn{5}{|l|}{ PSA, ng/mL } \\
\hline$<44$ & 0 & 0 & 1.00 (ref) & 0 \\
\hline$\geq 44$ & $0.30 \pm 0.15$ & $0.34 \pm 0.15$ & $1.40(1.05-1.89)$ & 1 \\
\hline Harrell's c-index & 0.7235 & $0.7173^{*}$ & & \\
\hline Optimism & & 0.006 & & \\
\hline
\end{tabular}




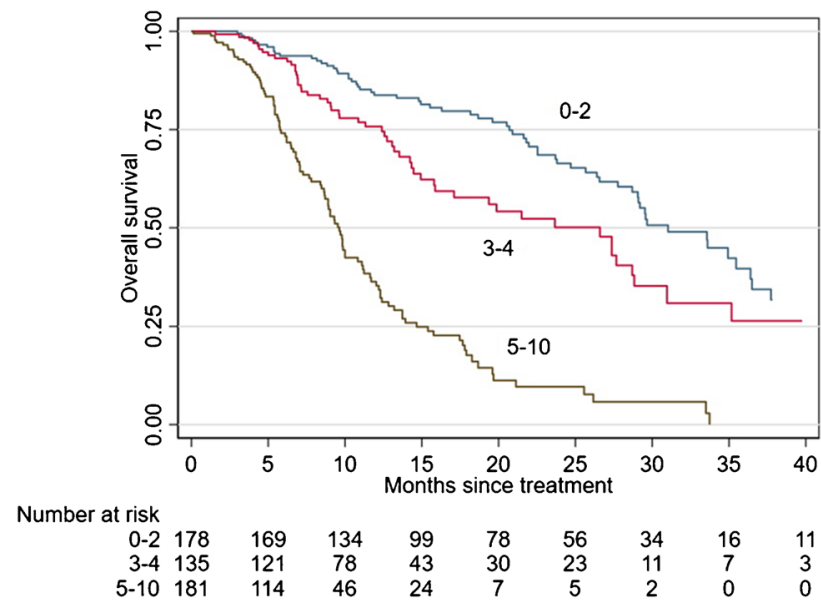

Fig. 3 Kaplan-Meier survival curves according to the BIO-Ra score. OS prediction according to the BIO-Ra score, categorizing patients into three prognostic groups (score $0-2,3-4$, and 5-10, showing mOS of 31.0, 26.6, and 9.6 months, respectively)

Given the consolidated role of cancer-related inflammation in tumorigenesis, prognosis, and response to oncological therapies [12], we extended our analysis to peripheral blood inflammatory indices. As a whole reflecting an inflamed state, these blood parameters have been investigated as potential prognostic and predictive factors in different settings [15]. In the last years, a few studies showed the predictive value of NLR in $\mathrm{mCRPC}$ patients treated with $\left[{ }^{223} \mathrm{Ra}\right] \mathrm{RaCl}_{2}$, related to the prediction of PFS and OS $[6,35]$. A recent monocentric study analyzed many inflammatory indices as prognostic factors in this clinical setting, confirming that NLR is the most accurate survival predictor among inflammatory indices [18].

Fig. 4 Emblematic cases belonging to the three BIO-Ra classes. Panels A-C represent the bone scans of three emblematic cases belonging to the three BIO-Ra classes (BIORa score 0-2, 3-4, and 5-10, respectively). The clinical and biochemical determinants of the BIO-Ra score are reported in each panel as well
However, most of the studies mentioned above remained inconclusive since none of these clinical or biochemical parameters has been validated as a unique and reliable selection tool. Consequently, in real-world clinical practice, we still encounter mCRPC patients with rapid disease progression after the $\left[{ }^{223} \mathrm{Ra}\right] \mathrm{RaCl}_{2}$ administration. With the BIO-Ra study, we developed a composite score combining performance status, tumor burden, and systemic inflammation, which can identify a subgroup of mCRPC patients who most likely benefit from $\left[{ }^{223} \mathrm{Ra}\right] \mathrm{RaCl}_{2}$ therapy. Its determinants are widely available in the clinical routine making the final prognostic score a broadly applicable tool for clinical practice with no additional costs. Emblematic cases belonging to the three prognostic categories are represented in Fig. 4.

Two previous studies have proposed integrating three prognostic biomarkers to obtain a 3-variable predicting score based on baseline ECOG PS, PSA, and $\mathrm{Hb}$ serum levels in mCRPC patients treated with $\left[{ }^{223} \mathrm{Ra}^{\mathrm{RaCl}} \mathrm{Ra}_{2}[8,45]\right.$. These studies were conducted on 92 and 430 patients, respectively. Compared with the larger study on 430 patients, the BIO-Ra study includes the ALP value and the number of bone metastases improving its reliability for stratifying the extent of the tumor burden compared to the sole PSA [46]. Moreover, the inclusion of NLR makes it able to describe at the same time the degree of systemic inflammation, which plays an essential role in the prognosis and response to therapies regardless of the extent of the tumor burden [18]. On the methodological ground, a robust approach based on bootstrap was used to define the optimal cut-off for each indices, to stabilize the c-index and the regression coefficients, and in general to consistently validate the obtained score.

Of note, even if prognostic, the baseline BIO-Ra score was not correlated with the biochemical response to therapy (measured as the dynamic change of ALP). Even if validated
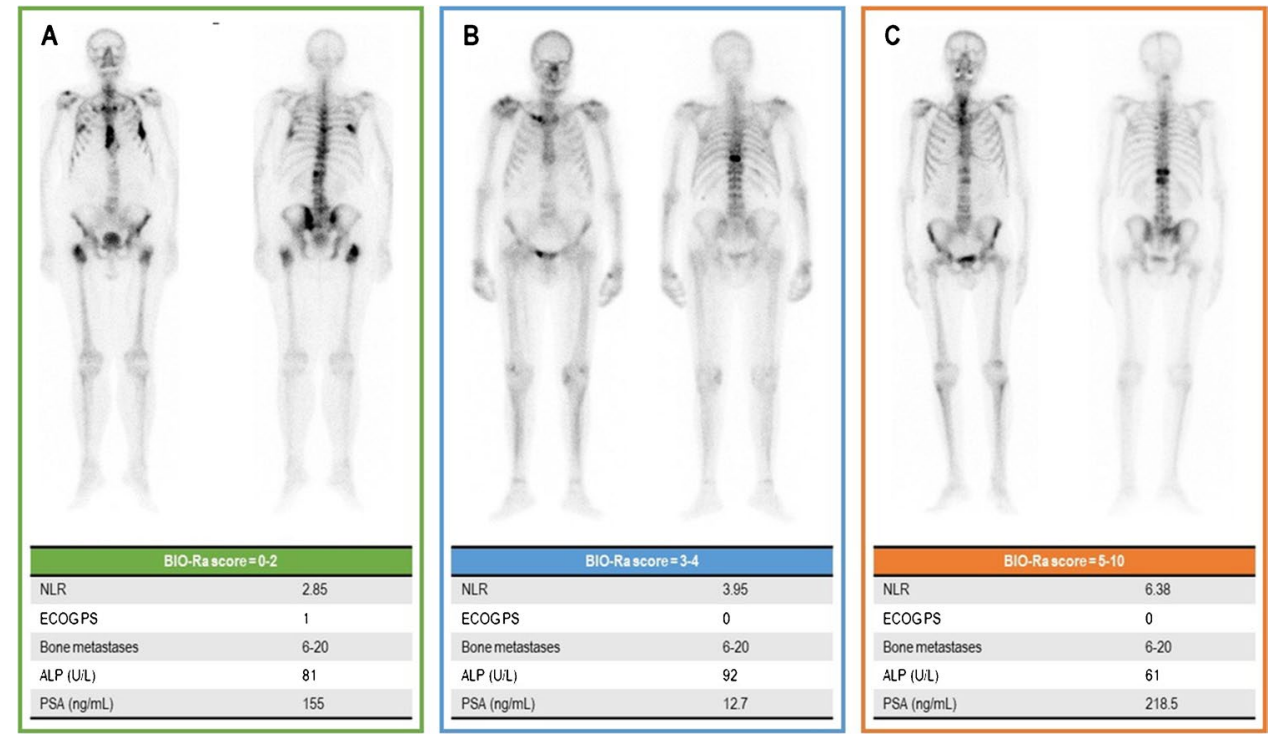
hematologic biomarkers for monitoring treatment response in mCRPC receiving $\left[{ }^{223} \mathrm{Ra}\right] \mathrm{RaCl}_{2}$ are currently lacking, a few studies reported that the ALP dynamic change might predict treatment benefit [28, 47, 48]. However, a post hoc analysis of the ALSYMPCA trial showed that while a reduction in ALP from baseline to week 12 significantly reduced the risk of death, proportional treatment effect values based on Cox regression models did not show the surrogacy for OS [28]. On these bases, it is currently not recommended to discontinue $\left[{ }^{223} \mathrm{Ra}\right] \mathrm{RaCl}_{2}$ therapy on the sole basis of dynamic ALP changes. It is reasonable that introducing a combinatory set of variables might better stratify treatment response concerning the only ALP change. Future studies might incorporate a combination of clinical and laboratory biomarkers when evaluating $\left[{ }^{223} \mathrm{Ra}\right] \mathrm{RaCl}_{2}$ treatment response. According to this, we are currently planning to analyze the prognostic power of the BIORa score dynamic change in $\left[{ }^{223} \mathrm{Ra}_{\mathrm{RaCl}}\right.$-treated patients.

The present study has some limitations. First, due to the multicentric nature of the study, even if made according to the current guidelines [19], the location of $\left[{ }^{223} \mathrm{Ra}\right]$ $\mathrm{RaCl}_{2}$ in the therapeutic sequence with the other life-prolonging agents for mCRPC was chosen by each participating center, thus potentially introducing biases. Second, these results are someway preliminary, as they would need validation on an independent patient population. Furthermore, a prospective randomized trial is needed to confirm the capability of the BIO-Ra score in identifying patients who would most benefit from $\left[{ }^{223} \mathrm{Ra}\right] \mathrm{RaCl}_{2}$ treatment, thus optimizing the patient's selection process. In particular, the preserved survival benefit of $\left[{ }^{223} \mathrm{Ra}\right] \mathrm{RaCl}_{2}$ treatment in the presence of higher BIO-Ra risk classes needs to be clarified. As a final consideration, the cohort of 519 mCRPC patients was exclusively treated with $\left[{ }^{223} \mathrm{Ra}\right] \mathrm{RaCl}_{2}$ plus androgen deprivation therapy. Thus, we cannot assume the generalizability of the BIO-Ra score's prognostic role to $\left[{ }^{223} \mathrm{Ra} \mathrm{RaCl}_{2}\right.$-based treatment combinations that are currently under investigation.

\section{Conclusion}

In the BIO-Ra study, we investigated the prognostic role of clinical factors and inflammatory indices and their combination in a prognostic score in mCRPC patients receiving [ $\left.{ }^{223} \mathrm{Ra}\right] \mathrm{RaCl}_{2}$ therapy. The BIO-Ra score allows an accurate prognostic stratification of mCRPC patients treated with $\left[{ }^{223} \mathrm{Ra}\right] \mathrm{RaCl}_{2}$, providing an easy and widely applicable tool for clinical practice at no additional costs. Future plans include the external validation of the prognostic value, the assessment of its predictivity, and its application in patients receiving $\left[{ }^{223} \mathrm{Ra} \mathrm{RaCl}_{2}\right.$-based treatment combinations.
Supplementary Information The online version contains supplementary material available at https://doi.org/10.1007/s00259-021-05550-6.

Author contribution Conceptualization: GF, SER, and MB. Data collection: MB, MID, VF, ELR, MM, VL, VM, AG, MLS, ML, LC, VL, PM, LC, MM, AS, GS, RPC, AS, GR, FM, and GDV. Statistical analysis: AS, and MP. Writing-original draft preparation: MB, SER, and VM. Writing-review and editing: all coauthors. Funding acquisition: n.a. All authors have read and agreed to the published version of the manuscript.

Funding Open access funding provided by Università degli Studi di Genova within the CRUI-CARE Agreement.

Data availability The data that support the findings of this study are available from the corresponding author upon reasonable request.

\section{Declarations}

Ethics approval The local ethical committees approved the retrospective multicentric study.

Consent to participate All patients signed a written informed consent at the time of radium-223 administration, encompassing the use of anonymized data for retrospective research purposes.

Conflict of interest The authors declare no competing interests.

Open Access This article is licensed under a Creative Commons Attribution 4.0 International License, which permits use, sharing, adaptation, distribution and reproduction in any medium or format, as long as you give appropriate credit to the original author(s) and the source, provide a link to the Creative Commons licence, and indicate if changes were made. The images or other third party material in this article are included in the article's Creative Commons licence, unless indicated otherwise in a credit line to the material. If material is not included in the article's Creative Commons licence and your intended use is not permitted by statutory regulation or exceeds the permitted use, you will need to obtain permission directly from the copyright holder. To view a copy of this licence, visit http://creativecommons.org/licenses/by/4.0/.

\section{References}

1. Keller ET, Brown J. Prostate cancer bone metastases promote both osteolytic and osteoblastic activity. J Cell Biochem. 2004;91:718-29.

2. McDougall JA, Bansal A, Goulart BH, McCune JS, Karnopp A, Fedorenko C, et al. The clinical and economic impacts of skeletal-related events among Medicare enrollees with prostate cancer metastatic to bone. Oncologist. 2016;21:320-6.

3. Harrison MR, Wong TZ, Armstrong AJ, George DJ. Radium-223 chloride: a potential new treatment for castration-resistant prostate cancer patients with metastatic bone disease. Cancer Manag Res. 2013;5:1-14.

4. Parker C, Nilsson S, Heinrich D, Helle SI, O’Sullivan JM, Fosså $\mathrm{SD}$, et al. Alpha emitter radium-223 and survival in metastatic prostate cancer. N Engl J Med. 2013;369:213-23.

5. Kluetz PG, Pierce W, Maher VE, Zhang H, Tang S, Song P, et al. Radium Ra 223 dichloride injection: U.S. Food and Drug Administration drug approval summary. Clin Cancer Res. 2014;20:9-14. 
6. Parikh S, Murray L, Kenning L, Bottomley D, Din O, Dixit S, et al. Real-world outcomes and factors predicting survival and completion of radium 223 in metastatic castrate-resistant prostate cancer. Clin Oncol (R Coll Radiol). 2018;30:548-55.

7. Wong WW, Anderson EM, Mohammadi H, Daniels TB, Schild SE, Keole SR, et al. Factors associated with survival following radium-223 treatment for metastatic castration-resistant prostate cancer. Clin Genitourin Cancer. 2017;15:e969-75.

8. Frantellizzi V, Farcomeni A, Follacchio GA, Pacilio M, Pellegrini R, Pani R, et al. A 3-variable prognostic score (3-PS) for overall survival prediction in metastatic castration-resistant prostate cancer treated with 223Radium-dichloride. Ann Nucl Med. 2018;32:142-8.

9. Den RB, George D, Pieczonka C, McNamara M. Ra-223 treatment for bone metastases in castrate-resistant prostate cancer: practical management issues for patient selection. Am J Clin Oncol. 2019;42:399-406.

10. EMA. EMA restricts use of prostate cancer medicine XOFIGO. Available online: https://www.ema.europa.eu/en/news/ema-restr icts-use-prostate-cancer-medicine-xofigo (accessed on 8 May 2021).

11. Kuppen MC, Westgeest HM, van der Doelen MJ, van den Eertwegh AJ, Coenen JL, Aben KK, et al. Real-world outcomes of radium-223 dichloride for metastatic castration resistant prostate cancer. Future Oncol. 2020;16:1371-84.

12. Hanahan D, Weinberg RA. Hallmarks of cancer: the next generation. Cell. 2011;144:646-74.

13 Templeton AJ, McNamara MG, Šeruga B, Vera-Badillo FE, Aneja $\mathrm{P}$, Ocaña A, et al. Prognostic role of neutrophil-to-lymphocyte ratio in solid tumors: a systematic review and meta-analysis. J Natl Cancer Inst. 2014;106:dju124.

14. Liu R, Zheng S, Yuan Q, Zhu P, Li B, Lin Q, et al. The prognostic significance of combined pretreatment fibrinogen and neutrophillymphocyte ratio in various cancers: a systematic review and meta-analysis. Dis Markers. 2020;2020:4565379.

15. Cupp MA, Cariolou M, Tzoulaki I, Aune D, Evangelou E, Berlanga-Taylor AJ. Neutrophil to lymphocyte ratio and cancer prognosis: an umbrella review of systematic reviews and meta-analyses of observational studies. BMC Med. 2020;18:360.

16. Guan Y, Xiong H, Feng Y, Liao G, Tong T, Pang J. Revealing the prognostic landscape of neutrophil-to-lymphocyte ratio and platelet-to-lymphocyte ratio in metastatic castration-resistant prostate cancer patients treated with abiraterone or enzalutamide: a meta-analysis. Prostate Cancer Prostatic Dis. 2020;23:220-31.

17. Man YN, Chen YF. Systemic immune-inflammation index, serum albumin, and fibrinogen impact prognosis in castration-resistant prostate cancer patients treated with first-line docetaxel. Int Urol Nephrol. 2019;51:2189-99.

18. Bauckneht M, Rebuzzi SE, Signori A, Donegani MI, Murianni V, Miceli A, et al. The prognostic role of baseline metabolic tumor burden and systemic inflammation biomarkers in metastatic castration-resistant prostate cancer patients treated with radium-223: a proof of concept study. Cancers (Basel). 2020;12:3213.

19. Poeppel TD, Handkiewicz-Junak D, Andreeff M, Becherer A, Bockisch A, Fricke E, et al. EANM guideline for radionuclide therapy with radium-223 of metastatic castration-resistant prostate cancer. Eur J Nucl Med Mol Imaging. 2018;45:824-45.

20. Heagerty PJ, Lumley T, Pepe MS. Time-dependent ROC curves for censored survival data and a diagnostic marker. Biometrics. 2000;56:337-44.

21. Cattaneo M, Malighetti P, Spinelli D. Estimating receiver operative characteristic curves for time-dependent outcomes: the stroccurve package. Stand Genomic Sci. 2017;17:1015-23.
22. Liu X. Classification accuracy and cut point selection. Stat Med. 2012;31:2676-86.

23. Liu X, Jin Z. Optimal survival time-related cut-point with censored data. Stat Med. 2015;34:515-24.

24. Kaplan EL, Meier P. Nonparametric estimation from incomplete observations. J Am Stat Assoc. 1958;53:457-81.

25. StataCorp. Stata statistical software: release 16. College Station: StataCorp LLC; 2019.

26. R Core Team. R: a language and environment for statistical computing. R Foundation for Statistical Computing, Vienna, Austria. URL https://www.R-project.org/. Accessed 1 Jun 2021.

27. Mehta HB, Mehta V, Girman CJ, Adhikari D, Johnson ML. Regression coefficient-based scoring system should be used to assign weights to the risk index. J Clin Epidemiol. 2016;79:22-8.

28. Sartor O, Coleman RE, Nilsson S, Heinrich D, Helle SI, O'Sullivan JM, et al. An exploratory analysis of alkaline phosphatase, lactate dehydrogenase, and prostate-specific antigen dynamics in the phase 3 ALSYMPCA trial with radium-223. Ann Oncol. 2017;28:1090-7.

29. Soloway MS, Hardeman SW, Hickey D, Raymond J, Todd B, Soloway S, et al. Stratification of patients with metastatic prostate cancer based on extent of disease on initial bone scan. Cancer. 1988;61:195-202.

30. De Vincentis G, Gerritsen W, Gschwend JE, Hacker M, Lewington V, O'Sullivan JM, et al. Advances in targeted alpha therapy for prostate cancer. Ann Oncol. 2019;30:1728-39.

31. McKay RR, Jacobus S, Fiorillo M, Ledet EM, Cotogna PM, Steinberger AE, et al. Radium-223 use in clinical practice and variables associated with completion of therapy. Clin Genitourin Cancer. 2017;15:e289-98.

32. Saad F, Carles J, Gillessen S, Heidenreich A, Heinrich D, Gratt $\mathrm{J}$, et al. Radium-223 and concomitant therapies in patients with metastatic castration-resistant prostate cancer: an international, early access, open-label, single-arm phase $3 \mathrm{~b}$ trial. Lancet Oncol. 2016;17:1306-16.

33. Naito M, Ukai R, Hashimoto K. Bone scan index can be a useful biomarker of survival outcomes in patients with metastatic castration-resistant prostate cancer treated with radium-223. Cancer Rep (Hoboken). 2019;2:e1203.

34. Prelaj A, Rebuzzi SE, Buzzacchino F, Pozzi C, Ferrara C, Frantellizzi V, et al. Radium-223 in patients with metastatic castrationresistant prostate cancer: efficacy and safety in clinical practice. Oncol Lett. 2019;17:1467-76.

35. Maruzzo M, Basso U, Borsatti E, Evangelista L, Alongi F, Caffo $\mathrm{O}$, et al. Results from a large, multicenter, retrospective analysis on radium 223 use in metastatic castration-resistant prostate cancer (mCRPC) in the Triveneto Italian Region. Clin Genitourin Cancer. 2019;17:e187-94.

36. Parimi S, Tsang E, Alexander A, Mckenzie M, Bachand F, Sunderland $\mathrm{K}$, et al. A population-based study of the use of radium 223 in metastatic castration-resistant prostate cancer: factors associated with treatment completion. Can Urol Assoc J. 2017;11:350-5.

37. De Luca R, Costa RP, Tripoli V, Murabito A, Cicero G. the clinical efficacy of radium-223 for bone metastasis in patients with castration-resistant prostate cancer: an Italian clinical experience. Oncology. 2018;94:161-6.

38. Vidal M, Delgado A, Martinez C, Correa JJ, Durango IC. Overall survival prediction in metastatic castration-resistant prostate cancer treated with radium-223. Int Braz J Urol. 2020;46:599-611.

39. Bauckneht M, Capitanio S, Donegani MI, Zanardi E, Miceli A, Murialdo R, et al. Role of baseline and post-therapy 18F-FDG PET in the prognostic stratification of metastatic castration-resistant prostate cancer (mCRPC) patients treated with radium-223. Cancers (Basel). 2019;12:31. 
40. Filippi L, Spinelli GP, Chiaravalloti A, Schillaci O, Equitani F, Bagni O. Prognostic value of 18F-Choline PET/CT in Patients with metastatic castration-resistant prostate cancer treated with radium-223. Biomedicines. 2020;8:555.

41. Roque V, Jessop M, Pereira L, Gape P, Dizdarevic S, Sousa E, et al. Bone scan index as metastatic bone disease quantifier and predictor of radium-223-dichloride biochemical response. Nucl Med Commun. 2019;40:588-96.

42. Frantellizzi V, Pani A, Ippoliti MD, Farcomeni A, Aloise I, Colosi $\mathrm{M}$, et al. Scintigraphic load of bone disease evaluated by DASciS software as a survival predictor in metastatic castration-resistant prostate cancer patients candidates to $223 \mathrm{RaCl}$ treatment. Radiol Oncol. 2019;54:40-7.

43. Bauckneht M, Bertagna F, Donegani MI, Durmo R, Miceli A, De Biasi V, et al. The prognostic power of 18F-FDG PET/CT extends to estimating systemic treatment response duration in metastatic castration-resistant prostate cancer (mCRPC) patients. Prostate Cancer Prostatic Dis. 2021. https://doi.org/10.1038/ s41391-021-00391-8.

44. Ricci M, Frantellizzi V, Bulzonetti N, De Vincentis G. Reversibility of castration resistance status after Radium-223 dichloride treatment: clinical evidence and review of the literature. Int J Radiat Biol. 2019;95:554-61.

45. Frantellizzi V, Monari F, Mascia M, Costa R, Rubini G, Spanu A, et al. Validation of the 3-variable prognostic score (3-PS) in
mCRPC patients treated with 223Radium-dichloride: a national multicenter study. Ann Nucl Med. 2020;34:772-80.

46. Akimoto S, Furuya Y, Akakura K, Shimazaki J, Ito H. Relationship between prostate-specific antigen, clinical stage, and degree of bone metastasis in patients with prostate cancer: comparison with prostatic acid phosphatase and alkaline phosphatase. Int $\mathbf{J}$ Urol. 1997;4:572-5.

47. Saad F, Gillessen S, Heinrich D, Keizman D, O'Sullivan JM, Nilsson S, et al. Disease characteristics and completion of treatment in patients with metastatic castration-resistant prostate cancer treated with radium-223 in an international early access program. Clin Genitourin Cancer. 2019;17:348-355.e5.

48. van der Doelen MJ, Stockhaus A, Ma Y, Mehra N, Yachnin J, Gerritsen WR, et al. Early alkaline phosphatase dynamics as biomarker of survival in metastatic castration-resistant prostate cancer patients treated with radium-223. Eur J Nucl Med Mol Imaging. 2021. https://doi.org/10.1007/s00259-021-05283-6.

Publisher's note Springer Nature remains neutral with regard to jurisdictional claims in published maps and institutional affiliations.

\section{Authors and Affiliations}

\section{Matteo Bauckneht ${ }^{1,2}$ (D) Sara Elena Rebuzzi ${ }^{3,4} \cdot$ Alessio Signori $^{2} \cdot$ Viviana Frantellizzi $^{5} \cdot$ Veronica Murianni $^{3,4}$. Elisa Lodi Rizzini ${ }^{6} \cdot$ Manlio Mascia $^{7} \cdot$ Valentina Lavelli $^{8} \cdot$ Maria Isabella Donegani ${ }^{1,2} \cdot$ Marta Ponzano $^{2}$. Angela Gaudiano $^{8} \cdot$ Maria Lina Stazza $^{9} \cdot$ Maria Licari $^{10} \cdot$ Letizia Cavallini $^{6,11} \cdot$ Viola Laghi $^{6,11} \cdot$ Luca Cindolo $^{12}$. Martina Maggi ${ }^{13}$. Alessandro Sciarra ${ }^{13}$. Paolo Mammucci ${ }^{8}$. Gianmario Sambuceti ${ }^{1,2} \cdot$ Renato Patrizio Costa $^{10}$. Angela Spanu ${ }^{9} \cdot$ Giuseppe Rubini $^{8} \cdot$ Fabio Monari $^{6} \cdot$ Giuseppe De Vincentis $^{5} \cdot$ Giuseppe Fornarini $^{3}$}

1 Nuclear Medicine, IRCCS Ospedale Policlinico San Martino, Genova, Italy

2 Department of Health Sciences (DISSAL), University of Genova, Genova, Italy

3 Medical Oncology Unit 1, IRCCS Ospedale Policlinico San Martino, Genova, Italy

4 Department of Internal Medicine and Medical Specialties, University of Genova, Genova, Italy

5 Department of Radiological Sciences, Oncology and Anatomical Pathology, Sapienza University of Rome, Rome, Italy

6 Radiation Oncology, IRCCS Azienda Ospedaliero-Universitaria Di Bologna, Bologna, Italy

7 Unit of Nuclear Medicine, Spirito Santo Hospital, Pescara, Italy
8 Nuclear Medicine Section, Interdisciplinary Department of Medicine, University of Bari "Aldo Moro", Bari, Italy

9 Unit of Nuclear Medicine, Department of Medical, Surgical and Experimental Sciences, University of Sassari, Sassari, Italy

10 Unit of Nuclear Medicine, Biomedical Department of Internal and Specialist Medicine, University of Palermo, Palermo, Italy

11 Department of Experimental Diagnostic and Specialty Medicine, Alma Mater Studiorum Bologna University, Bologna, Italy

12 Department of Urology, Villa Stuart Private Hospital, Rome, Italy

13 Department of Urology, Sapienza University of Rome, Policlinico Umberto I, Rome, Italy 